\title{
IME: um editor de ações e atividades de aprendizagem com apoio pedagógico flexível ao professor
}

\author{
Aladir Ferreira Silva Júnior ${ }^{12}$, Leandro Roberto Silva ${ }^{2}$, Clovis Torres Fernandes ${ }^{1}$ \\ ${ }^{1}$ Divisão de Ciência da Computação - Instituto Tecnológico de Aeronáutica (ITA) - \\ Laboratório de Aprendizagem e Interação (LAI) \\ CEP 12228-901 - São José dos Campos - SP - Brasil \\ 2 Coordenação de Informática - Instituto Federal de Educação, Ciência e Tecnologia de \\ Goiás (IFG) - Câmpus Jataí \\ CEP 75804-020 - Jataí - GO - Brasil \\ aladir@\{gmail.com, ita.br\}, leandroroberto.br@gmail.com, \\ clovistf@uol.com.br

\begin{abstract}
Resumo. Neste artigo se descreve o IME, um editor de ações e atividades de aprendizagem no padrão IMS Learning Design que tem como principal diferencial em relação a outros editores de mesmo fim, um apoio pedagógico flexível ao professor na estruturação de seu material didático digital. Além de apresentar as principais funcionalidades do IME se discute a importância de um software desse tipo no contexto educacional.
\end{abstract}

\section{Introdução}

Com o intuito de auxiliar, em maior ou menor grau, o professor na tarefa de estruturar material didático especificado no padrão IMSLD e seguindo uma abordagem pedagógica, diversos editores IMSLD (ELD) tem surgido. Os ELDs são ferramentas importantes pois podem permitir que um professor centrado em outros aprofundamentos teóricos aquém de teorias de aprendizagem e técnicas pedagógicas consagradas, possa se beneficiar destas na estruturação de seu material didático digital.

O apoio ao usuário quanto a parte pedagógica se dá por meio do fornecimento de um conjunto de estratégias a serem seguidas, chamado de modelo instrucional (MI). O MI pode ser representado computacionalmente usando o padrão IMSLD. O professor pode então seguir as orientações dadas pelo MI para estruturar seu material didático, transformando-o em uma Unidade de Aprendizagem (UoL, do original Unit of Learning), que pode ser uma lição, um curso que poderá ser disponibilizado aos usuários ou grupos de usuários de um Ambiente Virtual de Aprendizagem (AVA) ou ainda a usuários independentes por meio de um executor (player) stand-alone.

Apesar da diversidade de editores LD, constatam-se limitações nestes, especialmente quanto ao apoio aos usuários em relação à estruturação do material didático digital com base em técnicas pedagógicas ou no conjunto delas. Foram observadas as seguintes limitações: 
(i) a maioria dos ELDs não oferecem apoio ao professor na estruturação de seu material didático quanto aos modelos instrucionais. São exemplos o CopperAuthor, o Editor LD LAI (Fernandes et al., 2012), o LD Authoring Tool, o MOCOLADE, o MOT+LD, o Pathway ASK-LDT e o Reload; (ii) alguns ELDs tem sido desenvolvidos para atender a apenas um modelo instrucional (MI). Um exemplo é o editor WQE Editor (Camargo et al., 2010);

(iii) alguns ELDs oferecem ao professor vários MIs, porém não permitem seu uso de modo conjunto (intercalado) na estruturação de uma unidade de aprendizagem (e.g. um curso). São exemplos os editores CADMOS e Recourse; (iv) a maioria dos editores IMSLD não permitem reusar material já estruturado por outro editor, apesar de serem todos editores do padrão IMSLD;

(v) em uma pequena parcela dos ELDs que dispõe de auxílio ao professor com vários MIs e permitem intercalação de MIs, ainda é notada a falta de flexibilidade em não permitir a criação de novos MIs (Collage) ou ainda em relação a forma de intercalação possível entre os MIs, exigindo re-trabalho por parte do professor (LAMS e Open GLM Prolix). Além disso, (vi) apenas os editores Reload, Recourse e Collage conseguem importar material feito por outros ELDs sem maiores problemas.

O objetivo principal do editor IME, desenvolvido com base em um modelo de mesmo nome, é auxiliar em termos pedagógicos o professor na estruturação de seu material didático digital usando o padrão IMS Learning Design, superando as limitações do atual estado da arte da autoria de ações e atividades de aprendizagem em IMSLD. Os professores, especialistas em educação e demais envolvidos com ensino-aprendizagem formam o público-alvo do IME. Assim, pode-se dizer que o material a ser estruturado no IME pode atender a uma diversidade grande de público, níveis de ensino e disciplinas.

\section{Desenvolvimento}

O IME foi desenvolvido como uma aplicação $W e b$, portanto, cliente-servidor utilizando tecnologias atuais de desenvolvimento tais como a linguagem Java, a API Java Architecture for XML Binding (JAXB), o banco de dados relacional PostgreSQL, o servidor de aplicações Apache Tomcat, a linguagem HyperText Markup Language (HTML), a linguagem Java Server Pages (JSP), a linguagem eXtensible Markup Language (XML), a linguagem XML Schema Definition (XSD), a linguagem JavaScript com a biblioteca jQuery e a especificação Cascading Style Sheets (CSS).

Como processo de desenvolvimento adotou-se o Processo Unificado (UP, acrônimo do original Unified Process) baseando-se nos conceitos propostos em (Larman, 2007). O UP se constitui em um conjunto de atividades necessárias para transformar requisitos do usuário em sistema de software. Uma das vantagens do UP (a de ser incremental) foi explorada no desenvolvimento do IME. Mesmo sem estar com todo o software pronto foi possível utilizar as principais funcionalidades à medida que elas iam sendo adicionadas ao software.

A partir dos requisitos iniciais foi possível definir alguns papeis e gerar um artefato proposto pelo UP, o diagrama de casos de uso (vide Figura 1), que visa caracterizar o escopo do sistema. É possível perceber uma hierarquia dada aos usuários 
do IME, começando pelo usuário "Convidado". Todos os outros usuários vão herdando suas possibilidades de atuação. Assim o usuário "Administrador" pode realizar todas as atividades dentro do sistema IME.

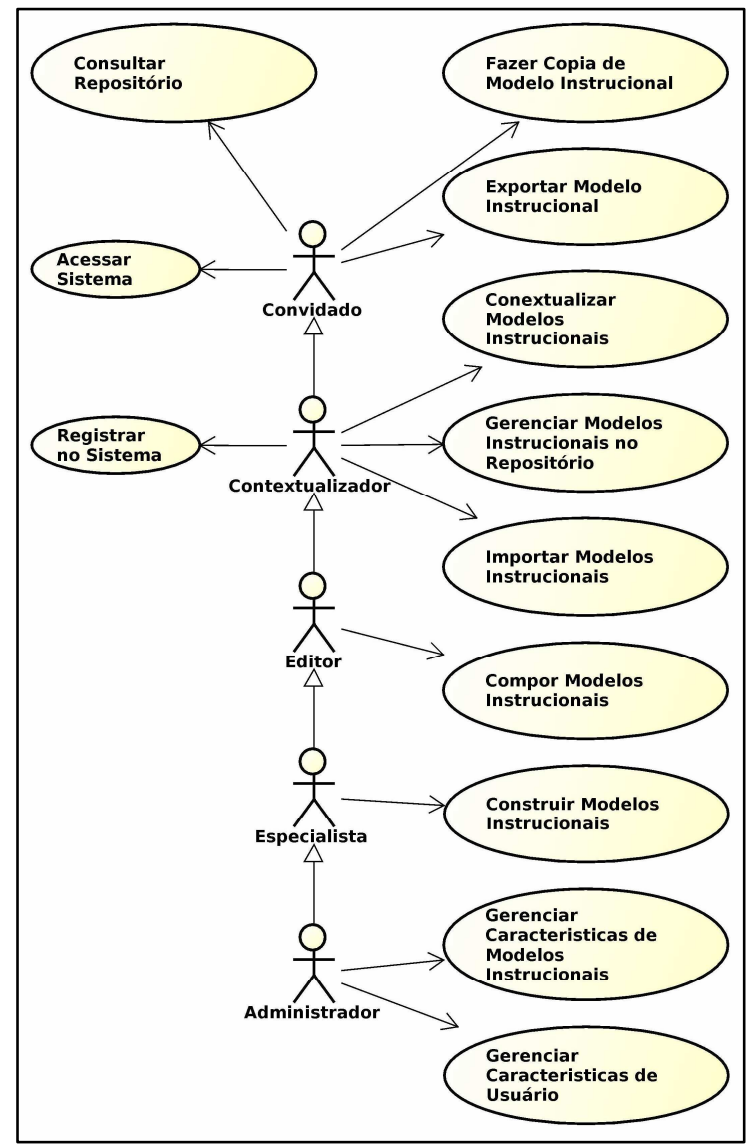

Figura 1 - Diagrama de Casos de Uso - IME

Para validação do IME se utilizou em uma primeira etapa um conjunto de simulações para mostrar de modo extensivo o uso de composições no IME. Foi desenvolvido a partir do algoritmo de composição do IME com o objetivo de gerar construções de MIs (estrutura básica com orientações ao professor a partir de técnicas pedagógicas) e UoLs (material didático já estruturado de acordo com um MI) de modo automático a partir de alguns parâmetros de entrada.

O simulador gera um conjunto de arquivos que podem ser importados no ambiente IME. Os MIs quando importados e disponibilizados no Workspace do IME, são editores LD específicos, com todas as funcionalidades necessárias para a geração de código IMSLD (UoLs).

Como segundo passo da validação está em curso um estudo do IME com a participação de 15 professores, sendo estes com atuação em cursos técnicos de nível médio, tecnológicos, de licenciatura e bacharelados do Instituto Federal de Educação, Ciência e Tecnologia de Goiás, Câmpus Jataí (IFG). Alguns desses professores (3) também atuam no Mestrado em Educação de Ciências e Matemática nesta mesma instituição. Na próxima seção se apresenta o IME com suas principais funcionalidades. 


\section{Apresentação do IME}

Nesta seção se apresenta o IME com suas principais funcionalidades. À medida do possível (espaço e clareza) são apresentadas screen shots das principais telas. Esta seção foi dividida em subseções, onde em cada subseção se apresenta uma funcionalidade do editor.

O IME tem licenciamento livre e está disponível para acesso em http://ime.learningdesign.com.br. Ao acessar este endereço por meio de um navegador Web, o usuário será conduzido para uma tela inicial com uma saudação e com a possibilidade de algumas operações. Ao clicar em "Acessar", vide Figura 2 (1), o usuário será conduzido a uma outra tela para informar suas credenciais de acesso, conforme pode ser visto na Figura 2 (2).

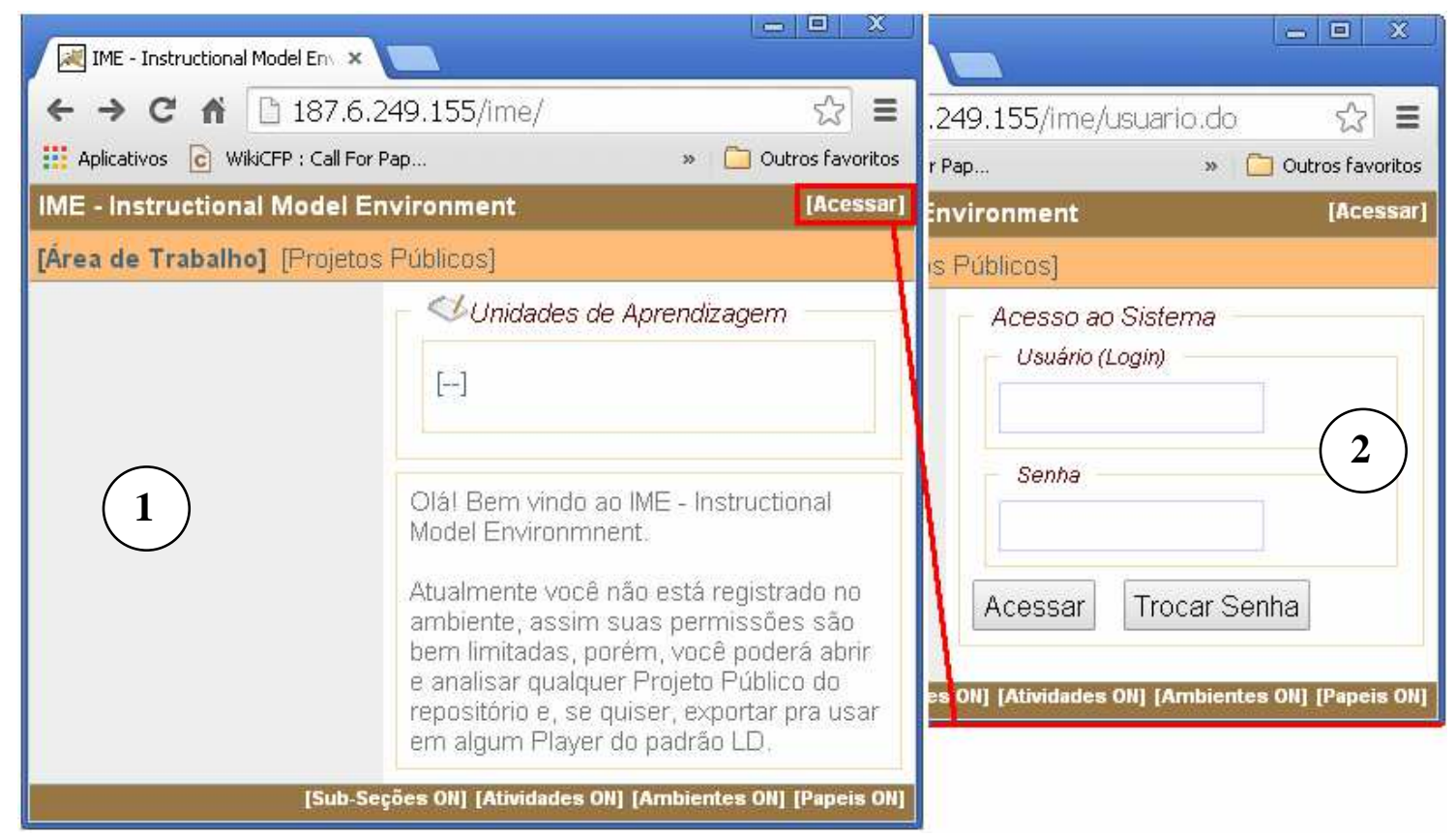

Figura 2 - Tela Inicial e Chamada de Login - IME

Ainda na tela inicial é possível ver os Projetos Públicos (MIs ou UoLs compartilhadas e de acesso livre). Ao clicar em Projetos Públicos qualquer usuário que acessou o IME pode adicionar um projeto à Área de Trabalho para visualização, no entanto as outras operações não estão disponíveis até que se tenha acesso com algum usuário registrado no IME.

Para avaliação, se disponibilizou no IME um acesso em três níveis. Em todos os níveis não é possível persistir no BD do IME os MIs ou UoLs criados, no entanto, é possível exportar e importar, o que permite trabalhar normalmente no ambiente, inclusive possibilitando exportar material pronto para ser colocado nos AVAs ou nos executores LD. Um vídeo demonstrativo pode ser acessado em http://ime.learningdesign.com.br/ime.mp4.

No primeiro nível, o usuário é um Contextualizador de MIs, ou seja, é possível utilizar MIs prontos para a criação de UoLs, no entanto não se permite criar MIs. Caso queira acessar como Contextualizador utilize usuário: $t c$ e senha: 123456. No segundo 
nível de acesso o usuário é um Editor e pode fazer composições (intercalações) de MIs prontos, bem como adicionar instruções às atividades dos MIs (futuras UoLs). Para acessar o IME como Editor utilize usuário: ted e senha: 123456. Por fim, no terceiro nível o usuário é um Especialista e tem a liberdade de criar um MI novo, compor MIs, adicionar e remover atividades e até mesmo contextualizar MIs, gerando UoLs. Para usar o IME como Especialista, acesse com o usuário: tesp e senha: $\mathbf{1 2 3 4 5 6 .}$

\subsection{Como criar um MI pelo IME?}

O usuário deve estar registrado no IME como Especialista e então terá acesso a Área de Trabalho que fornece um editor LD genérico com interface visual para a criação de um novo MI (vide Figura 3). Ao clicar no [+] o especialista já terá na área à esquerda da tela um MI novo (MI - Sem Título 0) com uma estrutura básica completa, apenas esperando por edição de seus componentes.

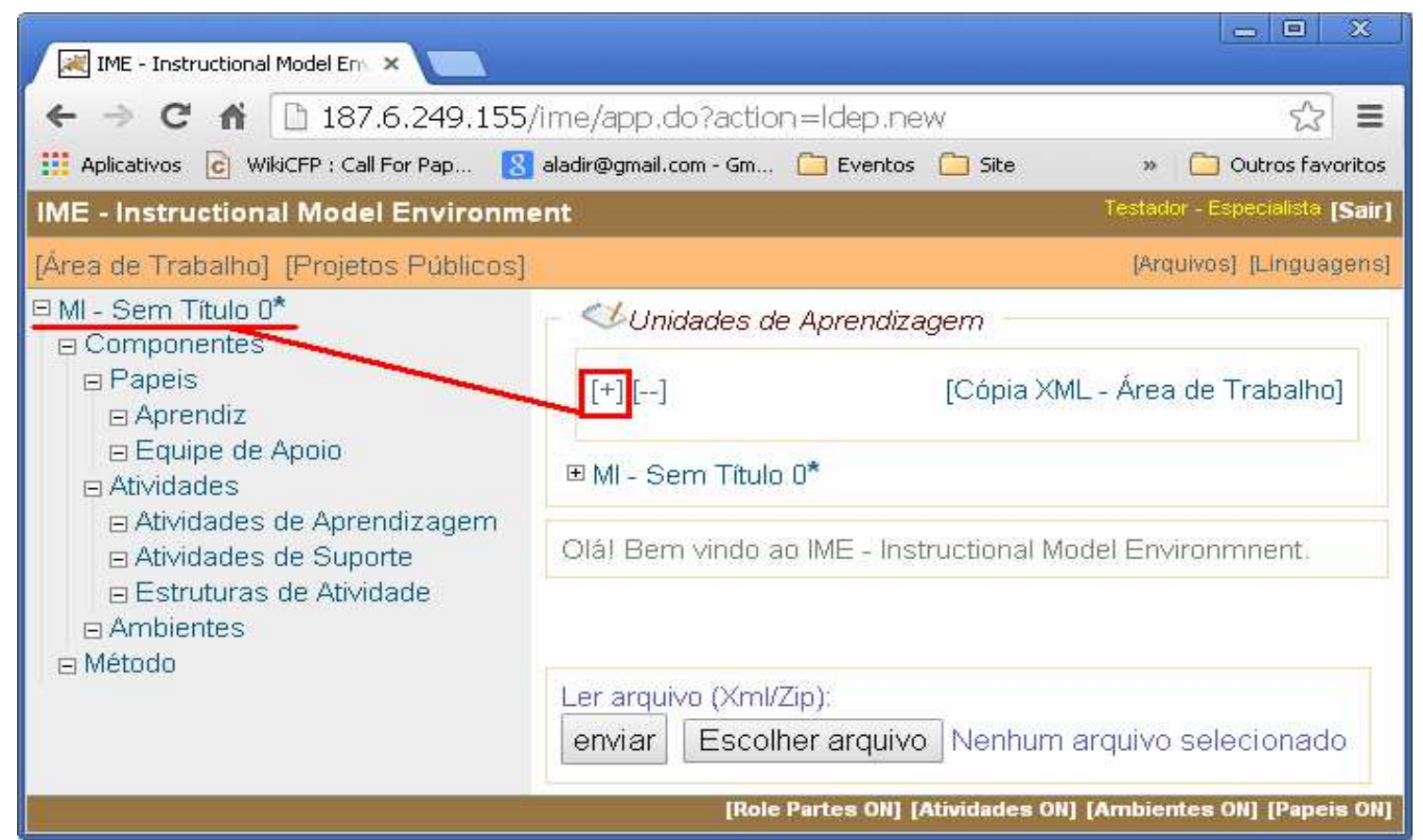

Figura 3 - Tela para criação de um novo MI no IME

O especialista deve então ir clicando nos itens do menu em árvore à esquerda para ter acesso a formulários específicos para cada um dos elementos, podendo assim adicionar, remover ou editar elementos. Ao se concluir esse processo já se terá pronto um MI que poderá ser exportado (que se constitui em um novo editor LD específico) ou ainda poderá ser disponibilizado na seção de Projetos Públicos para livre acesso a todos que usarem no IME.

\subsection{Como intercalar MIs pelo IME?}

Um recurso importante e que motivou a criação do ambiente IME é a possibilidade de que se faça a intercalação de um MI com outro MI (a especificação IMSLD não permite fazer isso!). Isso traz flexibilidade no momento em que se fornece apoio pedagógico ao professor na estruturação de seu material didático digital (criação 
de uma UoL, um curso ou uma lição, por exemplo). Assim o usuário do tipo Editor pode pegar dois MIs que estão no repositórios e adicioná-los ao Workspace.

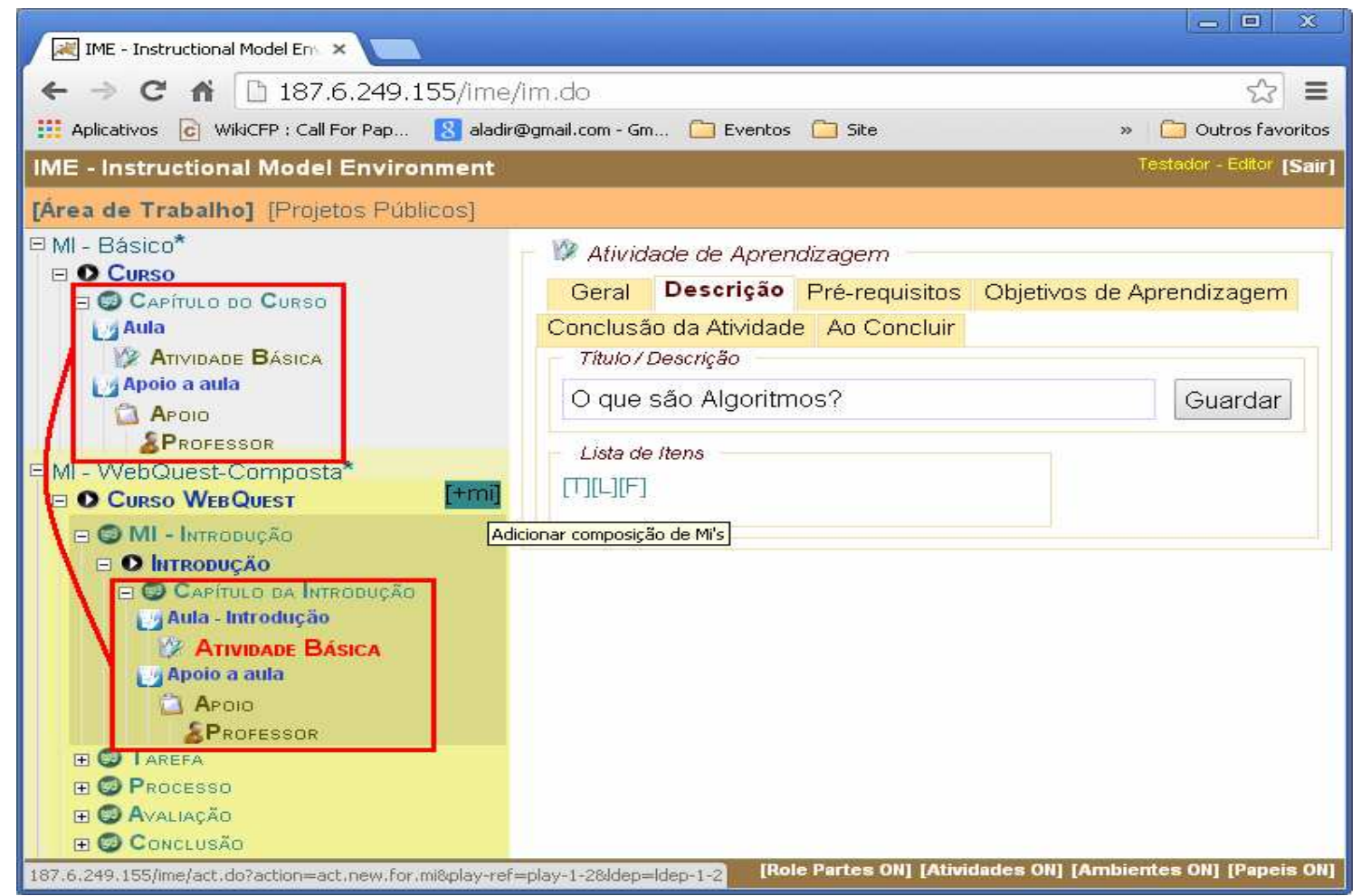

Figura 4 - Composição de MIs pelo IME

Pela Figura 4 é possível ver uma composição feita entre o "MI-Básico" e o "MIWebQuest-Composta". Ao clicar em [+mi] o usuário do tipo Editor pode incluir um "MI-Básico" na fase (ato ou elemento act) Introdução do "MI-WebQuest-Composta".

\subsection{Como criar uma UoL?}

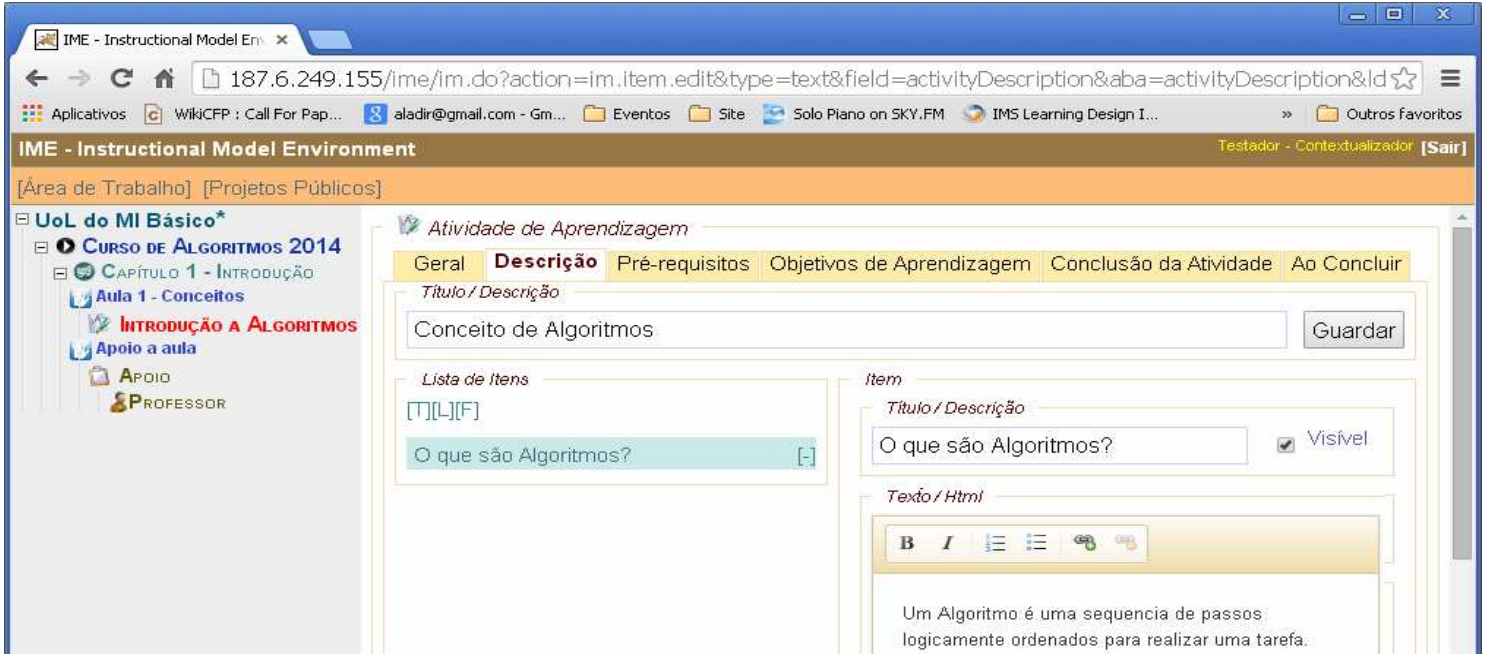

Figura 5 - Criação de uma Unidade de Aprendizagem (UoL) no IME 
Sendo uma UoL um MI contextualizado, é função de um usuário do tipo Contextualizador acessar o IME e adicionar os objetos de aprendizagem ou material didático relativos ao seu conteúdo, disciplina ou curso. Independentemente do conteúdo adicionado, o ambiente IME se comporta como um novo editor LD específico, fornecendo ao professor uma interface com um menu que segue o MI especificado. A Figura 5 mostra uma UoL para o tópico "Algoritmos" de um curso Técnico de Nível Médio em Informática, onde o professor está editando o elemento da aba "Descrição".

O próximo passo após se completar a inclusão de conteúdo na estrutura fornecida seguindo um MI é fazer a exportação para disponibilizar o material em um executor LD dentro ou fora de um Ambiente Virtual de Aprendizagem (e.g. Amadeus, Moodle, Tidia Sakai).

\subsection{Como importar e exportar MIs/UoLs?}

A importação é feita pela área de trabalho do IME (vide Figura 6) e pode se importar qualquer material estruturado no formato de UoL (encapsulado pelo padrão IMSCP, arquivo com extensão .zip) ou ainda com arquivo de manifesto independente (i.e. arquivo imsmanifest.xml). Ainda na Figura 6 se vê o arquivo .zip e seu conteúdo.

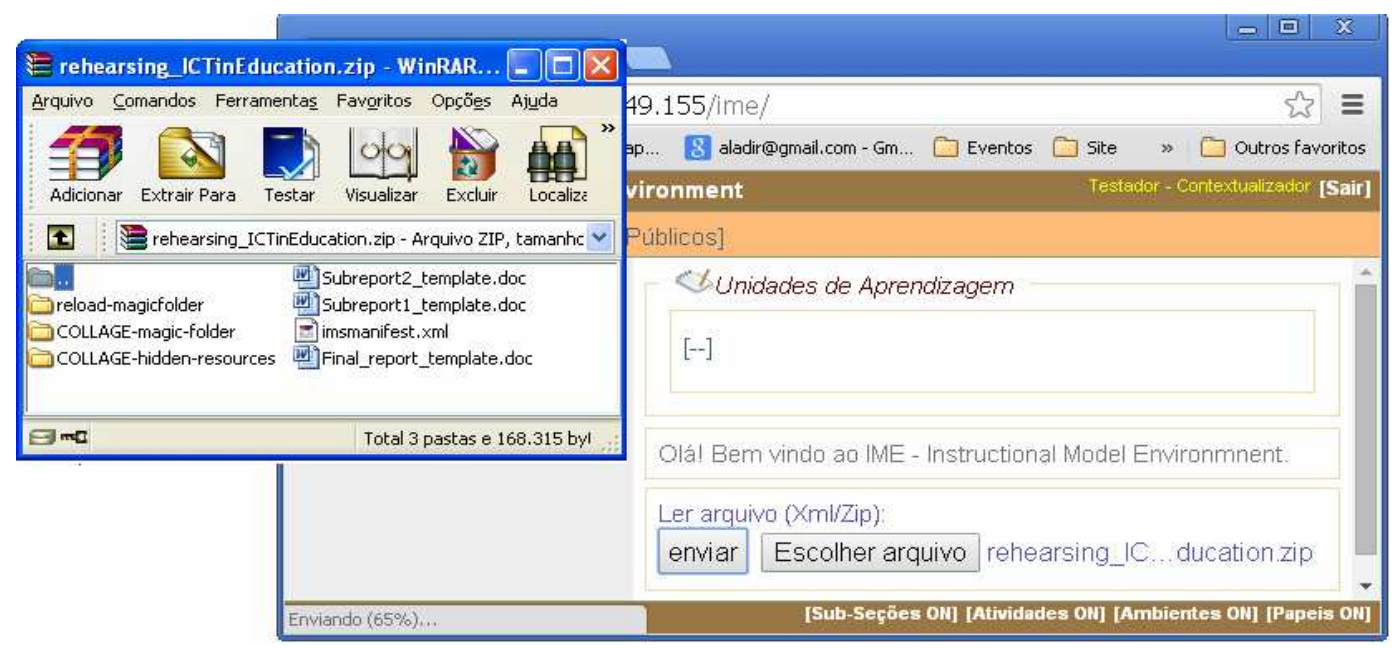

Figura 6 - Importação de Material seguindo o CLFP Jigsaw do Collage pelo IME

No editor Collage está disponível um MI denominado Jigsaw que é específico para a técnica pedagógica JigSaw ${ }^{1}$. Assim se importou um material estruturado com o uso desse MI e desenvolvido no Collage 2 . Como já abordado, o IME permite importar materiais estruturados por outros editores desde que estejam seguindo o padrão IMSLD. O MI importado pode ser visto na Figura 7 (1).

Para proceder à exportação no IME o usuário deverá escolher o projeto na área de menu em árvore (1) e acessar do lado direito a opção [Pacote Oficial IMSLD] (2), como se vê na Figura 7. Caso esteja faltando algum material ou elemento a definir será apresentado um relatório de pendências, caso contrário o usuário será conduzido a uma tela para escolher a pasta destino do arquivo .zip.

\footnotetext{
${ }^{1}$ Jigsaw Classroom - Overview of the Technique. Disponível em http://www.jigsaw.org/overview.htm.

${ }^{2}$ http://gsic.tel.uva.es/ dherleo/LD_example/rehearsing_ICTinEducation.zip
} 


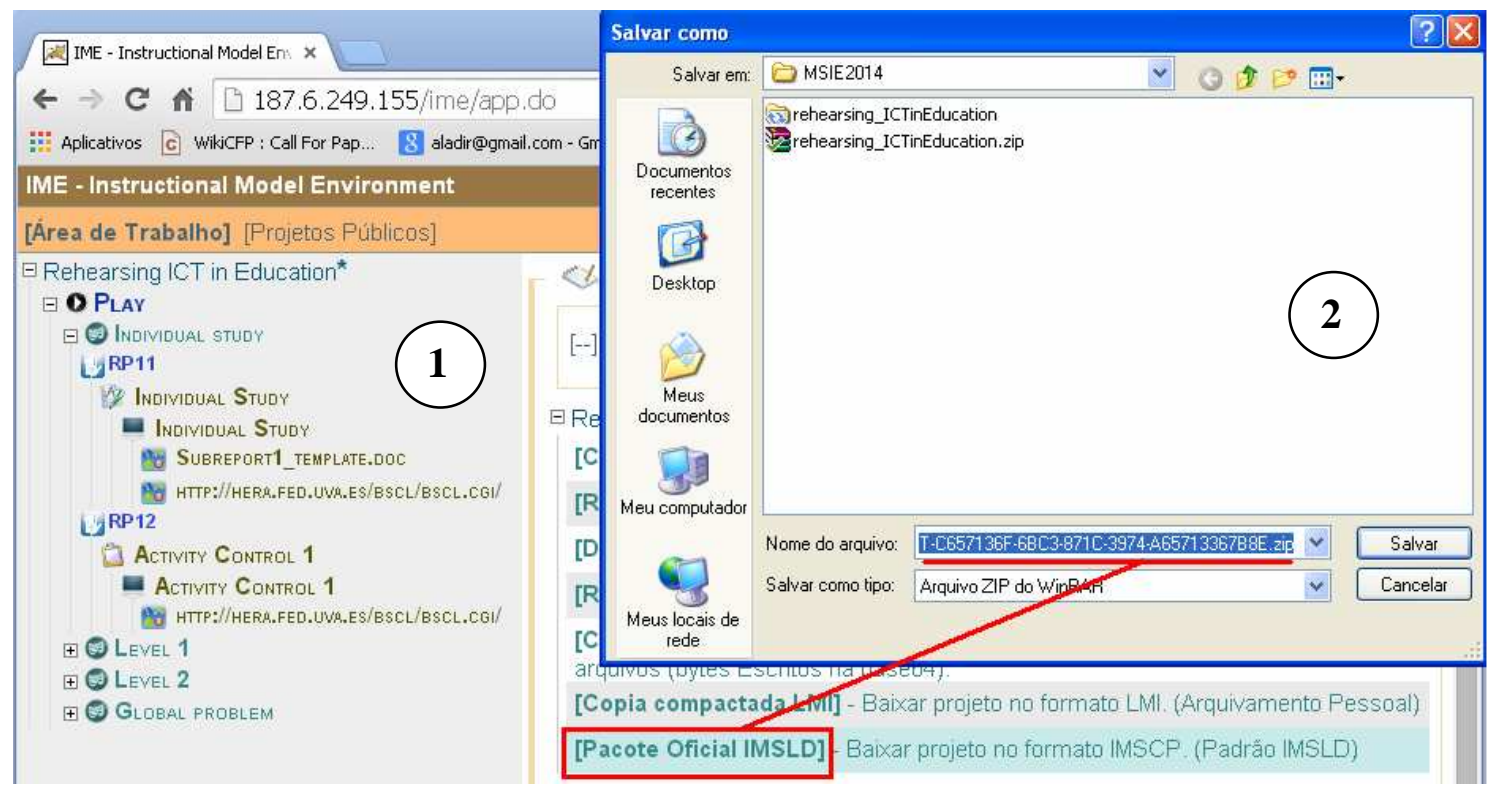

Figura 7 - (1) MI importado do Collage pelo IME. (2) Exportação de UoL no IME

\section{Considerações finais}

O IME é uma contribuição importante para o professor que deseja estruturar seu material didático digital usando o padrão IMS Learning Design. A flexibilidade em se poder intercalar os MIs para apoio pedagógico ao professor já é um quesito que justifica tal importância. A importação de um material desenvolvido em outro editor do padrão LD permite reúso e evita que o professor que já esteja acostumado com outras ferramentas possa usar seu material sem necessidade de adaptações.

O IME conseguiu por meio de simulação fazer a intercalação extensiva com sucesso de MIs tanto horizontalmente (um MI-pai e $k$ MIs-filhos), bem com em uma profundidade $n$. (e.g. $n=3$ equivale a se ter um MI-pai, um ou $k$ MIs-filhos e um ou $k$ MIs-netos). A validação feita com professores ainda não foi finalizada.

Como projetos futuros almeja-se a refatoração de algumas funcionalidades do IME (e.g. permitir um autocadastro de usuários, via Web), a disponibilização do editor para uso em larga escala e uma possível adequação do IME a outros padrões (e.g. IMS Rubric, IMS QTI, ADL SCORM, entre outros).

\section{Referências Bibliográficas}

Camargo, E. Z.; Fernandes, C. T. WQE um Editor de WebQuests Versátil. Anais do XXI Simpósio Brasileiro de Informática na Educação, 2010. João Pessoa-PB.

Fernandes, C. T.; Silva Júnior, A. F.; Varchavsky, M. Editor LD: Uma Ferramenta de Apoio à Autoria de Atividades de Aprendizagem no Padrão IMS Learning Design. II APPLETS - APplications to Provide LEarning and Teaching Support. Rio de Janeiro-RJ, 2012.

Larman, C. Utilizando UML e padrões: uma introdução à análise e ao projeto orientados a objetos e ao desenvolvimento iterativo. $3^{\text {a }}$. ed. Porto Alegre-RS. Bookman, 2007. 